\title{
Headache, cataract, and unilateral visual loss: unusual features of $D A R S 2$ variants in LBSL
}

\author{
Josef Finsterer ${ }^{\odot}$
}

Klinik Landstrasse, Messerli Institute, Vienna, Austria.

\section{Dear Editor,}

With interest we read the article by Cavusoglu et al. ${ }^{1}$ about a 12 year old male with bilateral congenital cataract, right-sided visual loss, recurrent frontal headache since age 9, paraspasticity, T2-hyperintensities of the corporis and genu callosum, posterior limb of internal capsule, inferior cerebellar peduncles, and mesencephalic portion of nucleus-V. ${ }^{1}$ Spinal magnetic resonance imaging (MRI) revealed T2-hyperintensities of the corticospinal tract, the spinocerebellar tracts, and the dorsal columns. ${ }^{1}$ Magnetic resonance spectroscopy (MRS) revealed a lactate peak. ${ }^{1}$ Based on these findings leukoencephalopathy with brainstem and spinal cord involvement and lactate elevation (LBSL) was suspected and confirmed by documentation of the variant c.455G $>$ T (p.C152F) (p.Cys152Phe)/c.22821_228-20delTTinsC in DARS2. ${ }^{1}$

We have the following comments and concerns.

Headache is an unusual phenotypic feature of LBSL. The authors should inform us about how the headache was classified (tensiontype, migraine, migraine-like, cluster). Since migraine-like headache is a frequent feature of mitochondrial disorders (MID) ${ }^{2}$, we should know if the headache was regarded as a feature of LBSL or a second problem independent of LBSL. The authors should also mention how the headache was treated and if the treatment was effective.

\section{Josef Finsterer}

fifigs1@yahoo.de

Received 15th October 2019, accepted 10th January 2021.
Congenital cataract is also unusual. Though congenital cataract has not been reported in association with LBSL, it is quite likely that it was a feature of the MID, since MIDs frequently go along with cataract in early ages. ${ }^{3,4}$ Cataract has been also reported in patients carrying IARS2 mutations. ${ }^{5}$ As DARS2, IARS2 encodes a tRNAsynthetase required for charging of $t R N A s$ with their cognate amino acid for translation.

Missing in this report is an extensive family history and genetic workup of first-degree relatives. Thus, it remains unclear if the DARS2 variant in the index case occurred spontaneously or was inherited. We should be informed if any of the first-degree relatives were clinically affected or not.

Bladder dysfunction in the form of a spastic bladder is an occasional feature of LBSL. ${ }^{6}$ It would have been interesting to find out if the index patient presented with bladder dysfunction and if steroids were beneficial as has been previously reported. ${ }^{5}$

Some of the LBSL patients present with double vision, spontaneous nystagmus, cognitive impairment $^{6}$, or developmental delay. ${ }^{7}$ Information concerning if any of these features were also found in the index patient or not should have been provided.

Extensive involvement of the spinal cord may not only occur in LBSL but has been also reported in other MIDs due to impaired aminotransferase ${ }^{8}$, such as ISCA2.

Unexplained remains the visual loss of the right eye. The authors did not inform us if the visual loss was due to complications from cataract surgery, damage of the optic nerve, or of the 
post-chiasmatic visual pathways. We should be informed about ophthalmologic investigations and the results of the visually-evoked potentials.

Overall, this interesting case could profit from the resolution of some shortcomings, such as a more detailed description of headache and congenital cataract, provision of an extensive family history, assessment of bladder function, comparison with rare features previously reported, and explanation of the unilateral visual loss.

Key words: myasthenia, seropositivity, acetylcholin-receptor antibodies, depression, quality of life, immunosuppression.

\section{REFERENCES}

1. Çavuşoğlu D, Olgaç-Dündar N, Öztekin Ö, Özdemir TR, Arıcan P, Gençpınar P. The first pediatric case of leukoencephalopathy with brainstem and spinal cord involvement and lactate elevation (LBSL) from Turkey. Turk J Pediatr 2018; 60: 216-220.

2. Finsterer J, Zarrouk-Mahjoub S. Headache in mitochondrial disorders. Clin Neurol Neurosurg 2018; 166: 44-49.
3. Liao SL, Huang SF, Lin JL, Lai SH, Chou YH, Kuo CY. Syndrome of mitochondrial myopathy of the heart and skeletal muscle, congenital cataract and lactic acidosis. Acta Paediatr Taiwan 2003; 44: 360364.

4. Choi BO, Hwang JH, Kim J, et al. A MELAS syndrome family harboring two mutations in mitochondrial genome. Exp Mol Med 2008; 40: 354-360.

5. Vona B, Maroofian R, Bellacchio E, et al. Expanding the clinical phenotype of IARS2-related mitochondrial disease. BMC Med Genet 2018; 19: 196.

6. Cheng FB, Shen PP, Zhou HW, Meng HM, Yang Y, Feng JC. Adult-onset leukoencephalopathy with brain stem and spinal cord involvement in Chinese Han population: a case report and literature review. Neurol India 2013; 61: 161-163.

7. Yahia A, Elsayed L, Babai A, et al. Intra-familial phenotypic heterogeneity in a Sudanese family with DARS2-related leukoencephalopathy, brainstem and spinal cord involvement and lactate elevation: a case report. BMC Neurol 2018; 18: 175.

8. Toldo I, Nosadini M, Boscardin C, et al. Neonatal mitochondrial leukoencephalopathy with brain and spinal involvement and high lactate: expanding the phenotype of ISCA2 gene mutations. Metab Brain Dis 2018; 33: 805-812.

\title{
Reply
}

\section{Response to "Headache, cataract, and unilateral visual loss: unusual features of DARS2 variants in LBSL"}

\author{
Dilek Çavuşoğlu ${ }^{1}$, Nihal Olgaç Dündar ${ }^{2}$
}

${ }^{1}$ Department of Pediatric Neurology, Faculty of Medicine, Afyonkarahisar Health Sciences University, Afyon; ${ }^{2}$ Department of Pediatric Neurology, Faculty of Medicine, İmir Katip Çelebi University, İzmir, Turkey.

\section{Dear Editor,}

This letter is a reply to the paper written by Josef Finsterer entitled "Headache, cataract, and unilateral visual loss: unusual features of DARS2 variants in LBSL" In his letter, Finsterer comments on a previous case presentation written by us entitled "The first pediatric case of leukoencephalopathy with brainstem and spinal cord involvement and lactate elevation
(LBSL) from Turkey". ${ }^{1}$ We would like to thank Finsterer for their comments and giving us the opportunity to further explain this interesting case.

The first question brought up concerned the type of headache experienced. We classified the headache as tension-type. There was no feature associated with migraine. We did not consider the headache to be a feature of LBSL. We firstly 
proposed life style modification and as the headaches were mild he was followed with non pharmacologic treatment.

The second comment raised concerned the patients congenital cataract and the fact that this is an unusual finding. We agree with this comment and the believe the cause of cataract in our patient to be associated with mitochondrial dysfunction. It is known that LBSL is a mitochondrial disease caused by homozygous or compound heterozygous mutation in the DARS2 gene encoding mitochondrial aspartyltRNA synthetase (mtAspRS). ${ }^{2}$ The visual loss of the right eye was informed to us by the family and was reported to be a consequence of the cataract operation performed in early ages. We are afraid there was no available results concerning recent ophthalmologic investigations or any result of the visually-evoked potentials. Moreover, our patient did not present with double vision, spontaneous nystagmus, cognitive impairment or developmental delay.

In their letter Finsterer discussed bladder dysfunction as an occasional feature of LBSL. Our case had no bladder dysfunction and therefore was not treated for this condition.
His family history did not include a similar case with features of LBSL. Genetic analysis was performed for variants in the parents. Our patient had a compound heterozygous mutation. As a result of these genetic analyses, it was shown that one of these mutations was taken from the mother and the other from the father.

We would like to thank you for the opportunity to respond to the issues addressed in Finsterers letter and to clarify aspects of our audit in relation to these concerns and believe all comments have been answered.

\section{REFERENCES}

1. Çavuşoğlu D, Olgaç-Dündar N, Öztekin Ö, Özdemir TR, Arıcan P, Gençpınar P. The first pediatric case of leukoencephalopathy with brainstem and spinal cord involvement and lactate elevation (LBSL) from Turkey. Turk J Pediatr 2018; 60: 216-220.

2. van der Knaap MS, van der Voorn P, Barkhof F, et al. A new leukoencephalopathy with brainstem and spinal cord involvement and high lactate. Ann Neurol 2003; 53: 252-258. 